\title{
Viabilidade da irrigação do meloeiro com águas salinas em diferentes fases fenológicas
}

\author{
Feasibility of irrigation of musk melon with salinity water in different phenological stages
}

\author{
Francisco de Queiroz Porto Filho ${ }^{1}$ José Francismar de Medeiros ${ }^{2}$ Eduardo Rodrigues de Sousa Neto ${ }^{3}$ \\ Hans Raj Gheyi ${ }^{4}$ José de Arimatea de Matos ${ }^{2}$
}

\section{RESUMO}

Com o objetivo de estudar os efeitos da aplicação de águas de irrigação de diferentes salinidades no rendimento do melão irrigado por gotejamento e de associar a produção obtida com o custo da água utilizada, desenvolveu-se este trabalho em Mossoró-RN. Águas de diferentes salinidades $\left(S_{1}=0,6, S_{2}=1,9, S_{3}=3,2\right.$ e $\left.S_{4}=4,5 d \mathrm{~d} \mathrm{~m}^{-1}\right)$, utilizadas de forma incremental em três estádios de desenvolvimento ou sem variar durante o ciclo da cultura, formaram dez tratamentos arranjados em blocos inteiramente casualizados com quatro repetições. $O$ uso de águas salinas por longos períodos afetou a produção de melão. Substituições tardias na salinidade da água tenderam a não exercer efeito significativo sobre a produção do meloeiro. O tratamento irrigado com a água de menor salinidade durante todo ciclo apresentou, simultaneamente, o maior custo com água de irrigação e o maior lucro na produção de melão.

Palavras-chave: Cucumis melo L., estresse salino, produção, custo da água, lucro.

\section{ABSTRACT}

This study was carried out in Mossoró, RN, Brazil, to evaluate the effects of different irrigation water salinity levels on yield of drip irrigated melon, and to relate yield with the cost of water. The waters of different salinities $\left(S_{1}=0.6, S_{2}=1.9\right.$, $S_{3}=3.2$ e $S_{4}=4.5 d S \mathrm{~m}^{-1}$ ) were used both in incremental way in three different phenological stages and without replacement during the crop cycle totalizing ten treatments arranged in a completely randomized block design with four repetitions. The use of saline waters without substitutions affected melon production. The treatments irrigated with low salinity water presented simultaneously the higher cost of irrigation water and higher profits of melon cultivation.
Key words: Cucumis melo L., saline stress, yield, cost of water, profit.

\section{INTRODUÇÃO}

A salinização causa efeitos negativos nas plantas pela diminuição do potencial osmótico da solução do solo e/ou pelos efeitos de íons específicos. As espécies e cultivares se comportam diferentemente à salinidade do solo, tolerando até um certo valor, sem reduzir o seu rendimento potencial, denominado salinidade limiar (SL). Com a salinidade a partir da SL a produtividade passa a diminuir. A tolerância das culturas aos sais é afetada por vários fatores, incluindo-se aí o estádio de desenvolvimento, a duração da exposição, as condições ambientais, propriedades do solo, o tipo e intensidade do manejo (MAAS, 1990).

O meloeiro apresenta grande variação no nível de tolerância à salinidade, variando tanto entre cultivares quanto em relação às condições ambientais e de manejo (PIZARRO, 1990; FRANCOIS \& MAAS, 1993). Normalmente, a redução de rendimento do melão ocorre pela diminuição no peso de fruto (MENDLINGER \& PASTERNAK, 1992; FRANCO et al., 1997). A tolerância do melão à salinidade não guarda muita relação entre os primeiros estádios de desenvolvimento e os últimos. Muitos trabalhos têm avaliado a sua tolerância à salinidade, nas diversas fases fenológicas

\footnotetext{
${ }^{1}$ Departamento de Ciências Ambientais, Universidade Federal Rural do Semi Árido (UFERSA), CP 137, 59625-900, Mossoró, RN, Brasil. E-mail: porto@ufersa.edu.br. Autor para correspondência.

${ }^{2}$ Departamento de Ciências Ambientais, UFERSA, Mossoró, RN, Brasil.

${ }^{3}$ Autônomo, Mossoró, RN, Brasil.

${ }^{4}$ Departamento de Engenharia Agrícola, Universidade Federal de Campina Grande, Campina Grande, PB, Brasil.
} 
(FRANCO et al., 1993; BOTÍA et al., 1998). Alguns trabalhos verificaram que os efeitos inibitórios da salinidade na cultura do melão diminuem à medida que a salinidade é imposta mais tarde, e concluíram que águas salobras podem ser usadas com o mínimo de perdas se a concentração de sais e a duração de exposição forem cuidadosamente monitoradas (MENDLINGER \& PASTERNAK, 1992; CARVAJAL et al., 1998; AMOR et al., 1999).

Na região do Pólo Agrícola Mossoró/Açu, no Estado do Rio Grande do Norte, a maior demanda por água para irrigação tem obrigado à utilização de águas com salinidade mais elevada. Segundo MEDEIROS (1992), MARTINS (1993), OLIVEIRA \& MAIA (1998), embora a maioria das fontes de água apresente boa qualidade, existem águas de qualidade inferior nessa região que, no entanto, podem ser utilizadas na irrigação. Na Chapada do Apodi, as águas subterrâneas são provenientes de dois tipos de aqüíferos, o Arenito Açu, com poços profundos $( \pm 1000 \mathrm{~m})$ e água de boa qualidade, com condutividade elétrica (CEa) em torno de $0,6 \mathrm{dS} \mathrm{m}^{-1}$, entretanto, exigindo alto investimento para sua captação e o Calcário Jandaíra, com poços rasos ( $\pm 100 \mathrm{~m})$, com menor custo de implementação do poço e de captação, mas com CEa variando de 1,2 a $4,5 \mathrm{dS} \mathrm{m}^{-1}$, rica $\mathrm{em} \mathrm{Ca}^{++}$e $\mathrm{HCO}_{3}^{-}$. Neste caso, a utilização da água fica condicionada à tolerância das culturas, à salinidade e ao manejo da irrigação com vistas ao controle da salinização do solo. Salienta-se que os métodos de irrigação por gotejamento são os mais adequados ao uso e manejo de águas salinas, por manterem a umidade do solo sempre elevada, não permitindo que a concentração de sais chegue a níveis críticos (DASBERG \& BRESLER, 1985; PIZARRO, 1990).

O objetivo do trabalho foi de estudar os efeitos de aplicação de águas de irrigação com diferentes níveis de salinidade, usadas durante todo o ciclo ou de forma incremental nos diferentes estádios de desenvolvimento do melão, visando verificar a possibilidade da substituição de águas menos salinas por de maior salinidade no meloeiro; proceder análise de custo da água versus produção, com intuição de maximizar o uso de águas salinas, usando-se como estratégia o incremento da salinidade da água de irrigação ao longo de ciclo da cultura.

\section{MATERIAL E MÉTODOS}

O trabalho foi composto por dois experimentos com o meloeiro (Cucumis melo L.), var. Inodorus, cv. AF646 (frutos com casca de coloração amarela), realizados na Fazenda Santa Júlia (latitude $5^{\circ}$ $02^{\prime} 0,02^{\prime}$ ' S; longitude $37^{\circ} 22^{\prime} 33,6^{\prime \prime} \mathrm{W}$ Gr. e altitude de 60 m), em Mossoró-RN. O clima do local, segundo Köppen é do tipo BSwh', isto é, seco, muito quente e com estação chuvosa no verão atrasando-se para o outono, apresentando temperatura média anual de $27,4^{\circ} \mathrm{C}$, precipitação pluviométrica anual bastante irregular, com média de $673,9 \mathrm{~mm}$, e umidade relativa do ar de $68,9 \%$ (CARMO FILHO \& OLIVEIRA, 1989). O solo foi classificado como Latossolo Vermelho Eutrófico argissólico, textura média (EMBRAPA, 1999), fase caatinga hiperxerófila e relevo plano (SUDENE, 1968). A tabela 1 apresenta a análise química e física por camada de solo.

Tabela 1 - Análise química e física por camada de solo

\begin{tabular}{|c|c|c|c|c|c|c|c|c|c|c|}
\hline \multirow{2}{*}{ Prof } & \multirow{3}{*}{ Água } & \multicolumn{3}{|l|}{$\mathrm{pH}$} & \multicolumn{4}{|c|}{ Complexo sortivo } & \multirow{3}{*}{$\begin{array}{c}\mathrm{P} \\
\left(\mathrm{mg} \mathrm{dm} \mathrm{dm}^{-3}\right)\end{array}$} & \multirow{3}{*}{$\begin{array}{c}\mathrm{CE}_{1: 2} \\
\left(\mathrm{dS} \mathrm{m}^{-1}\right)\end{array}$} \\
\hline & & $\mathrm{KCl}$ & $\mathrm{CaCl}_{2}$ & $\mathrm{Ca}$ & $\mathrm{Mg}$ & K & $\mathrm{Na}$ & $\mathrm{Al}$ & & \\
\hline$(\mathrm{cm})$ & & & & \multicolumn{5}{|c|}{$\left(\mathrm{cmol}_{\mathrm{c}} \mathrm{dm}^{-3}\right)$} & & \\
\hline $0-15$ & 6,8 & 5,7 & 6,2 & 2,50 & 0,60 & 0,30 & 0,27 & 0 & 53 & 0,15 \\
\hline $15-30$ & 6,5 & 5,5 & 6,0 & 2,50 & 0,60 & 0,34 & 0,25 & 0 & 40 & 0,09 \\
\hline $30-45$ & 5,2 & 4,3 & 4,9 & 1,60 & 1,10 & 0,31 & 0,28 & 0,10 & 10 & 0,14 \\
\hline \multirow[t]{3}{*}{$45-60$} & 4,9 & 4,1 & 4,6 & 1,20 & 0,90 & 0,25 & 0,23 & 0,20 & 2 & 0,08 \\
\hline & \multicolumn{3}{|c|}{ Fração granulométrica $\left(\mathrm{g} \mathrm{kg}^{-1}\right)$} & \multirow{2}{*}{\multicolumn{2}{|c|}{$\begin{array}{c}\text { Classe } \\
\text { Textural* }\end{array}$}} & \multicolumn{3}{|c|}{ Umidade $\left(\mathrm{cm}^{3} \mathrm{~cm}^{-3}\right)$} & \multicolumn{2}{|c|}{ Densidade $\left(\mathrm{kg} \mathrm{dm}^{-3}\right)$} \\
\hline & Argila & Silte & Areia & & & $\theta_{\operatorname{cc}(9,8 \mathrm{~Pa})}$ & & $\theta_{\mathrm{pmp}}$ & Dg & $\mathrm{Dp}$ \\
\hline $0-15$ & 120,6 & 172,2 & 707,2 & & & 0,15 & & 0,06 & 1,57 & 2,67 \\
\hline $15-30$ & 139,8 & 160,2 & 700,0 & & & 0,15 & & 0,06 & 1,54 & 2,72 \\
\hline $30-45$ & 200,5 & 211,6 & 587,9 & & & 0,24 & & 0,12 & 1,43 & 2,72 \\
\hline $45-60$ & 200,3 & 181,2 & 618,5 & & & 0,24 & & 0,12 & 1,50 & 2,68 \\
\hline
\end{tabular}

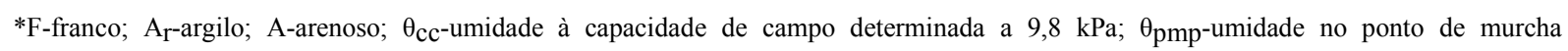
permanente determinado a $1500 \mathrm{kPa}$; Dg-densidade global; Dp-densidade de partícula. 
Os experimentos foram conduzidos nos períodos de 11 de outubro a 20 de dezembro de 2001 (Exp. I) e de 11 de setembro a 18 de novembro de 2002 (Exp. II). Aplicaram-se águas de irrigação, com quatro níveis de salinidade, durante todo o ciclo e de forma incremental, ao se substituírem águas de menor salinidade por de maior salinidade, em três diferentes estádios de desenvolvimento da cultura. As características químicas das águas de irrigação utilizadas estão na tabela 2. Foram empregados 10 tratamentos, compostos pelas seqüências: $\mathrm{S}_{1}, \mathrm{~S}_{1}$ e $\mathrm{S}_{1}$; $\mathrm{S}_{2}, \mathrm{~S}_{2}$ e $\mathrm{S}_{2} ; \mathrm{S}_{3}, \mathrm{~S}_{3}$ e $\mathrm{S}_{3} ; \mathrm{S}_{4}, \mathrm{~S}_{4}$ e $\mathrm{S}_{4} ; \mathrm{S}_{1}, \mathrm{~S}_{1}$ e $\mathrm{S}_{2} ; \mathrm{S}_{1}, \mathrm{~S}_{1}$ e $\mathrm{S}_{3}$; $\mathrm{S}_{1}, \mathrm{~S}_{1}$ e $\mathrm{S}_{4} ; \mathrm{S}_{1}, \mathrm{~S}_{2}$ e $\mathrm{S}_{2} ; \mathrm{S}_{1}, \mathrm{~S}_{3}$ e $\mathrm{S}_{3}$ e $\mathrm{S}_{1}, \mathrm{~S}_{4}$ e $\mathrm{S}_{4}$, denominados, respectivamente, de tratamentos $\mathrm{T}_{1}$ a $\mathrm{T}_{10}$, sendo as salinidades das águas $(\mathrm{S})$ de cada uma dessas seqüências correspondentes, respectivamente, aos níveis aplicados nos períodos do plantio até o aparecimento das flores femininas (30 dias após semeadura - DAS), do aparecimento das flores femininas até completo crescimento dos frutos (50 DAS) e deste até a maturação (colheita).

Os 10 tratamentos foram dispostos em blocos inteiramente casualizados, com quatro repetições, em 40 parcelas de $36 \mathrm{~m}^{2}$ (6 x 6m). Cada parcela foi composta por três fileiras de plantas espaçadas de $2 \mathrm{~m}$; na fileira foi utilizado espaçamento de 0,38 e $0,12 \mathrm{~m}$ em forma alternada, com uma planta por cova, resultando em uma população de plantio de 20.000 plantas ha-1 ${ }^{-1}$ Assumiu-se como área útil, de cada parcela, as 20 plantas situadas no centro da fileira central. $\mathrm{O}$ Experimento II foi uma repetição do I, utilizando-se, exatamente o mesmo local, tratamentos e delineamento experimental.

A área experimental foi irrigada por gotejamento, através de quatro sistemas de irrigação independentes. Os gotejadores utilizados foram do tipo auto-compensante, com vazão nominal de $2,3 \mathrm{~L} \mathrm{~h}^{-1}$, espaçados $0,50 \mathrm{~m}$; avaliados no campo, apresentaram vazões médias, coeficientes de variação de vazão e de uniformidade de vazão para, início do Exp. I e final dos Experimentos I e II, de: $2,17 \mathrm{~L} \mathrm{~h}^{-1}, 7 \%$ e 0,$93 ; 2,20 \mathrm{~L} \mathrm{~h}^{-1}$, $10 \%$ e 0,89 e $2,30 \mathrm{~L} \mathrm{~h}^{-1}, 6 \%$ e 0,93 , respectivamente.
As lâminas de irrigação foram determinadas a partir de estimativas da evapotranspiração da cultura utilizando-se o método de Penman-Monteith, proposto pela FAO (ALLEN et al., 1998) e ajustadas com base no monitoramento da umidade do solo com o uso de tensiômetros em duas repetições do tratamento $T_{1}$, adotando-se uma fração de lixiviação de 0,10 . O Kc basal adotado para as fases intermediária e final, correspondeu, respectivamente, a 1,0 e 0,7. No Exp. I, as lâminas de irrigação aplicadas nos períodos de 0 a 30,31 a 50 e 51 a 70 DAS foram, respectivamente, de 108,136 e $101 \mathrm{~mm}$. No período entre um experimento e outro (264 dias), a precipitação pluviométrica foi de $1228,00 \mathrm{~mm}$ e foram aplicados $381 \mathrm{~mm}$ de irrigação suplementar em cultura intercalar de milho, usando-se a água $\mathrm{S}_{3}$. No Exp. II, as lâminas de irrigação aplicadas nos períodos de 0 a 30, 31 a 50 e 51 a 68 DAS foram, respectivamente, de 95,132 e $85 \mathrm{~mm}$.

Os valores médios de temperatura média (Tmed), máxima (Tmax) e mínima (Tmin), umidade relativa (UR), velocidade do vento a $10 \mathrm{~m}(\mathrm{~V})$, insolação (I) e ETo para os Experimentos I e II foram de 28,9; 35,0 e $24,2^{\circ} \mathrm{C} ; 65,5 \% ; 5,5 \mathrm{~m} \mathrm{~s}^{-1} ; 9,1 \mathrm{~h}$ e $6,6 \mathrm{~mm} \mathrm{~d}^{-1}$ e de 28,4 ; 35,1 e $23,2^{\circ} \mathrm{C} ; 62,8 \% ; 6,0 \mathrm{~m} \mathrm{~s}^{-1} ; 10,4 \mathrm{~h}$ e $7,1 \mathrm{~mm} \mathrm{~d}^{-1}$, respectivamente.

Em ambos os experimentos, as adubações foram realizadas de acordo com a análise de solo da área experimental, atendendo-se às exigências nutricionais da cultura, conforme VIVANCOS (1996) e distribuídas ao longo do ciclo, de conformidade com LIMA (2001). O controle de ervas daninhas foi realizado por capinas manuais. Os frutos defeituosos, tipo "cabacinha" e doentes, foram desbastados. O controle das principais pragas e doenças comuns na região foi preventivo, através do uso de defensivos agrícolas.

A colheita foi realizada em três épocas, a intervalo de três dias, iniciando-se quando a maioria dos frutos se encontrava no ponto de colheita e se estendendo por seis dias. Em cada época, foram colhidos apenas os frutos com colorações predominantemente amarela, passíveis à

Tabela 2 - Composição química das águas com quatro níveis de salinidade (S) utilizadas no trabalho

\begin{tabular}{|c|c|c|c|c|c|c|c|c|c|c|c|}
\hline Água & $\mathrm{CE}$ & $\mathrm{pH}$ & $\mathrm{Ca}$ & $\mathrm{Mg}$ & $\mathrm{Na}$ & $\mathrm{K}$ & $\mathrm{CO}_{3}$ & $\mathrm{HCO}_{3}$ & $\mathrm{Cl}$ & $\mathrm{SO}_{4}$ & RAS \\
\hline \multicolumn{3}{|c|}{$\left(\mathrm{dS} \mathrm{m} \mathrm{m}^{-1}\right)$} & \multicolumn{8}{|c|}{$\left(\mathrm{mmol}_{\mathrm{c}} \mathrm{L}^{-1}\right)$} & $\left(\mathrm{mmol}_{\mathrm{c}} \mathrm{L}^{-1}\right)^{1 / 2}$ \\
\hline${ }^{1} \mathrm{~S}_{1}$ & 0,6 & 7,40 & 1,83 & 1,79 & 2,96 & 0,54 & 0,74 & 3,13 & 1,67 & Aus & 2,20 \\
\hline${ }^{2} \mathrm{~S}_{2}$ & 1,9 & 7,26 & 6,90 & 2,68 & 8,64 & 0,37 & 0,42 & 4,63 & 12,23 & Pres & 3,95 \\
\hline${ }^{3} \mathrm{~S}_{3}$ & 3,2 & 7,07 & 13,43 & 3,83 & 15,96 & 0,14 & 0,00 & 6,56 & 25,86 & Pres & 5,43 \\
\hline${ }^{4} \mathrm{~S}_{4}$ & 4,5 & 7,07 & 13,43 & 3,83 & 23,86 & 0,14 & 0,00 & 6,56 & 33,76 & Pres & 8,12 \\
\hline
\end{tabular}

${ }^{1}$ água de poço do aqüífero Arenito Açu, prof. de $1000 \mathrm{~m} ;{ }^{2}$ água obtida pela mistura de $\mathrm{S}_{1}$ com $\mathrm{S}_{3}$; ${ }^{3}$ água de poço do aqüífero Calcário Jandaíra, prof. de $80 \mathrm{~m} ;{ }^{4}$ preparada pela adição de $\mathrm{NaCl}$ a água $\mathrm{S}_{3}$, 
comercialização. Nos Experimentos I e II as colheitas foram realizadas, respectivamente, a 64, 67 e 70 DAS e a 62, 65 e 68 DAS. Os frutos foram contados, pesados e classificados para os mercados interno e externo, determinando-se a produção comercial e total.

As características de produção foram avaliadas, pela análise conjunta dos dois experimentos abrangendo os tratamentos $\mathrm{T}_{1} \mathrm{aT}_{10}$ no esquema fatorial $3 \times 3+1$, sendo três épocas para iniciar o uso de salinidade superior a $\mathrm{S}_{1}(0,30$ e 50 DAS $)$ e três níveis de salinidade $\left(\mathrm{S}_{2}, \mathrm{~S}_{3}\right.$ e $\left.\mathrm{S}_{4}\right)$ mais o nível $\mathrm{S}_{1}$ iniciado no tempo 0 (tratamento $\mathrm{T}$-adicional), conforme em AMOR et al. (1999), procedendo-se as análises de variância e de regressão, adotando-se probabilidade de até 0,05 . Utilizando-se os resultados de produção comercial, analisou-se o lucro de agricultura irrigada, através da equação 1, apresentada por BERNARDO (1998).

$$
\mathrm{Lw}=\mathrm{YPy}-(\mathrm{WPw}+\mathrm{C})
$$

Onde: Lw - lucro da agricultura irrigada (RS ha $\left.{ }^{-1}\right)$; Y - produção comercial $\left(\mathrm{Mg} \mathrm{ha}^{-1}\right)$; Py - preço do

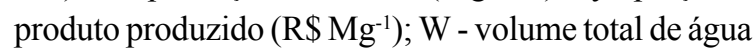
aplicada $\left(\mathrm{m}^{3} \mathrm{ha}^{-1}\right) ; \mathrm{Pw}$ - custo do fator água $\left(\mathrm{R} \$ \mathrm{~m}^{-3}\right)$ e C - custeio da produção sem considerar o fator água (R\$ $\left.\mathrm{ha}^{-1}\right)$.

A partir do preço das águas dos poços raso $\left(\mathrm{S}_{2}\right.$ a $\left.\mathrm{S}_{4}\right)$ e profundo $\left(\mathrm{S}_{1}\right)$, estimados na Fazenda Santa Júlia com os custos da potência consumida na sucção da água e manutenção do sistema de cada um dos poços, determinou-se o custo do fator água, através da média ponderada dos volumes aplicados com cada um dos tipos de águas, nas diversas fases, ao longo do ciclo da cultura nos dois experimentos. As águas $\mathrm{S}_{2}$ a $\mathrm{S}_{4}$, por serem provenientes de poços rasos, foram consideradas com o mesmo custo.

\section{RESULTADOS E DISCUSSÃO}

Na análise de variância, verificou-se que o aumento da salinidade das águas e do tempo de uso destas na irrigação do melão, diminuíram significativamente as produções comercial e total. As médias obtidas no Exp. II foram estatisticamente superiores àquelas alcançadas no primeiro experimento, nas duas variáveis avaliadas (Tabela 3).

As equações de regressão linear para produção comercial com incrementos na salinidade da água aos 0, 30 e 50 DAS, foram todas significativas (Figura 1). Avaliando-se a produção total, pode-se constatar que, nas equações de regressão linear, os efeitos foram significativos para incrementos na salinidade da água de irrigação aos 0 e 30 DAS, sendo o incremento aos 50 DAS não significativo (Figura 1). Observando-se esta figura, afirma-se que o efeito restritivo da salinidade da água de irrigação, tanto na produção comercial como na total, é mais acentuado quando a água salina é utilizada mais cedo. Além disso, considerando-se tempos iguais de uso, a perda no rendimento da cultura foi maior quando se utilizou água de irrigação mais salina, concordando com o observado por AMOR et al. (1999), FRANCO et al. (1993) e FRANCO et al. (1997) e abordado por FRANCOIS \& MAAS (1993).

Essas afirmações se tornam mais claras ao se observar a figura 1, construída com base nos rendimentos relativos de produção comercial e total, considerando a produção com o uso de água $\mathrm{S}_{1}$ igual a $100 \%$. Nas equações do gráfico de produção comercial, verifica-se que, ao se utilizar a água $\mathrm{S}_{4}$ a partir de 0 DAS, o meloeiro produzirá $67,4 \%$ e, se for empregada apenas a partir dos 50 DAS, a produção comercial da cultura passará a ser de 90,2\%. Considerando-se tempos iguais de uso de águas de salinidades diferentes, podese afirmar que, se a partir de 30 DAS, for utilizada na irrigação do melão água $\mathrm{S}_{4}$ e $\mathrm{S}_{2}$, a produção da cultura será de 78,5 e 92,8\%, respectivamente. Esses resultados confirmam o verificado por AMOR et al. (1999) e MENDLINGER \& PASTERNAK (1992) ao afirmarem que, quanto maior a salinidade da água e o tempo de uso da mesma na irrigação, maior a perda no rendimento da cultura. Nas equações obtidas a partir dos rendimentos relativos, houve redução na produção comercial da cultura de 8,$35 ; 5,52$ e 2,50\%, por acréscimo de $1 \mathrm{dS} \mathrm{m}^{-1}$ na salinidade da água de irrigação aos 0,30 e 50 DAS, respectivamente, enquanto que, na produtividade total do meloeiro, as diminuições foram de 6,0, 4,6 e 0,9\%, respectivamente. Através desses valores se confirma, ainda, que quando se retarda o aumento na salinidade da água, menor é a perda no rendimento da cultura do melão.

Os volumes médios acumulativos de água aplicados nos dois experimentos, nos períodos de 0 a 30,31 a 50 e 51 a 68 ou 70 DAS foram, respectivamente, 1014,1338 e $927 \mathrm{~m}^{3} \mathrm{ha}^{-1}$. Os preços da água estimados na Fazenda Santa Júlia, dos poços raso e profundo 
Tabela 3 - Resumo de ANOVA e médias de produção comercial (Pcom) e total (Ptotal) do meloeiro submetido a quatro níveis de salinidade da água de irrigação e três tempos de início da salinização em análise conjunta dos Experimentos I e II. Mossoró, 2001 e 2002.

\begin{tabular}{|c|c|c|c|c|c|c|c|}
\hline Variáveis & & Pcom & Ptotal & Variáveis & & Pcom & Ptotal \\
\hline FV & GL & \multicolumn{2}{|c|}{ Estatística F (cor.) } & FV & GL & \multicolumn{2}{|c|}{ Estatística F (cor.) } \\
\hline Bloco & 3 & $8,49 * *$ & $10,95 * *$ & $\mathrm{~S} /$ Tempo 30 & 3 & $2,83 *$ & $4,73 * *$ \\
\hline Experimento(E) & 1 & $16,85^{* *}$ & $44,91 * *$ & Reg. Lin. & 1 & $7,52 * *$ & $12,19 * *$ \\
\hline Salinidade (S) & 3 & $12,74 * *$ & $13,93 * *$ & Reg. Quad. & 1 & $0,58^{\mathrm{ns}}$ & $1,91^{\mathrm{ns}}$ \\
\hline Tempo & 2 & $7,28 * *$ & $12,16^{* *}$ & $\mathrm{~S} /$ Tempo 50 & 3 & $4,00 *$ & $2,64^{\mathrm{ns}}$ \\
\hline Bloco*E & 3 & $0,42^{\mathrm{ns}}$ & $0,74^{\mathrm{ns}}$ & Reg. Lin. & 1 & $6,97 * *$ & $2,04^{\mathrm{ns}}$ \\
\hline$E^{*} \mathrm{~S}$ & 3 & $0,98^{\mathrm{ns}}$ & $0,80^{\mathrm{ns}}$ & Reg. Quad. & 1 & $4,59 *$ & $0,024^{\mathrm{ns}}$ \\
\hline E*Tempo & 2 & $0,93^{\mathrm{ns}}$ & $0,15^{\mathrm{ns}}$ & QM Resíduo & 54 & 25,2324 & 15,8747 \\
\hline $\mathrm{E}^{*} \mathrm{~S} *$ Tempo & 6 & $0,40^{\mathrm{ns}}$ & $0,57^{\mathrm{ns}}$ & $\mathrm{CV}(\%)$ & & 15,93 & 9,92 \\
\hline S*Tempo & 6 & $1,55^{\mathrm{ns}}$ & $2,36^{*}$ & \multirow{2}{*}{\multicolumn{2}{|c|}{ Experimento }} & \multicolumn{2}{|c|}{ Média } \\
\hline $\mathrm{S} /$ Tempo 0 & 3 & $9,02 * *$ & $11,28 * *$ & & & $\mathrm{Mg} \mathrm{ha}^{-1}$ & $\mathrm{Mg} \mathrm{ha}^{-1}$ \\
\hline Reg. Lin. & 1 & $26,77 * *$ & $32,27 * *$ & I & & $30,16 \mathrm{~b}$ & $38,11 \mathrm{~b}$ \\
\hline Reg. Quad. & 1 & $0,15^{\mathrm{ns}}$ & $0,004^{\mathrm{ns}}$ & II & & $34,37 \mathrm{a}$ & $43,56 \mathrm{a}$ \\
\hline
\end{tabular}

$\left({ }^{\mathrm{ns}}\right)$ não significativos a $0,05,(*)$ significativo a 0,05 e $(* *)$ a 0,01 de probabilidade, pelo teste $\mathrm{F}$

foram, respectivamente, em $\mathrm{R} \$ \mathrm{~m}^{-3}$, de 0,05 e 0,16 . Considerando-se que as águas $\mathrm{S}_{2} \mathrm{a}_{4}$ são provenientes de poços rasos, verificou-se que os custos do uso da água $\mathrm{S}_{1}$ durante o ciclo da cultura $\left(\mathrm{T}_{1}\right)$ foi de $\mathrm{RS}$ $508,33 \mathrm{ha}^{-1}$ e quando a salinidade foi incrementada aos 50,30 e 0 DAS, os custos foram de $\mathrm{R} \$ 413,75 ; \mathrm{R} \$ 277,23$ e R $\$ 173,82 \mathrm{ha}^{-1}$, respectivamente, correspondendo a 81,$4 ; 54,5$ e $34,2 \%$ do custo de $\mathrm{T}_{1}$ (Tabela 4 ).

As produções comerciais relativas, estimadas a partir das equações apresentadas na figura 1, para incrementos na salinidade aos 50, 30 e 0 DAS variaram, respectivamente, de 87,3 a 98,8; 78,1 a 90,1 e 66,1 a $88,7 \%$, do valor verificado com o uso da água menos salina durante todo o ciclo da cultura $(100 \%)$. O menor valor desses três intervalos de variação referese ao uso da água $\mathrm{S}_{4}$ durante todo o ciclo e o maior ao uso de $\mathrm{S}_{2}$ a partir de 50 DAS, após se usar $\mathrm{S}_{1}$ (Tabela 4).

O lucro da cultura do melão irrigado ( $\mathrm{Lw}$ ) determinado com a equação 1 , em valores absolutos e percentuais em relação ao tratamento $\mathrm{T}_{1}$, utilizando-se o custo da água por tratamento, conforme explicado no parágrafo anterior, está apresentado na tabela 4 . Apesar de se verificar em $\mathrm{T}_{1}$, o maior custo da água, por se utilizar apenas água de poço profundo $\left(\mathrm{S}_{1}\right)$ durante todo o ciclo, foi nele que se obteve o maior Lw, devido à sua maior produção comercial. Com o incremento da salinidade aos 50 e 30 DAS, o Lw variou, respectivamente, de 98,5 a $68,0 \%$ e de 77,7 a $46,0 \%$, em relação ao $\mathrm{Lw}$ de $\mathrm{T}_{1}$, quando as águas utilizadas também oscilaram de $\mathrm{S}_{2}$ a $\mathrm{S}_{4}$. Com o uso de $\mathrm{S}_{2}, \mathrm{~S}_{3}$ e $\mathrm{S}_{4}$ durante todo o ciclo da cultura, obteve-se um Lw, respectivamente, de 75,7; 45,8 e 15,9\%, em relação ao de $T_{1}\left(S_{1} S_{1} S_{1}\right)$.

Salienta-se que os custos da água considerados se referem aos custos variáveis, com base na energia de bombeamento e na manutenção dos poços e bombas, não se considerando os custos fixos (investimentos) de perfuração e instalação das bombas que, para os poços profundos, podem variar de 40 a 60 vezes os dos poços rasos. Portanto, seria necessária uma análise mais ampla e profunda incluindose os custos fixos de cada poço, com vistas à determinação da área de plantio mínimo a compensar a instalação de poço profundo.

\section{CONCLUSÕES}

Aumentos na salinidade da água de irrigação reduzem as produções comercial e total do meloeiro. Quanto mais próximo do início do ciclo cultural do meloeiro se usarem águas salobras na irrigação, maior será a redução da produção comercial e total de frutos. Apesar do custo da água de irrigação proveniente de poços profundos e com menor salinidade ter sido mais elevado, o uso desta água apresentou maior lucro que o uso de águas salobras provenientes de poços rasos.

Ciência Rural, v.36, n.2, mar-abr, 2006. 


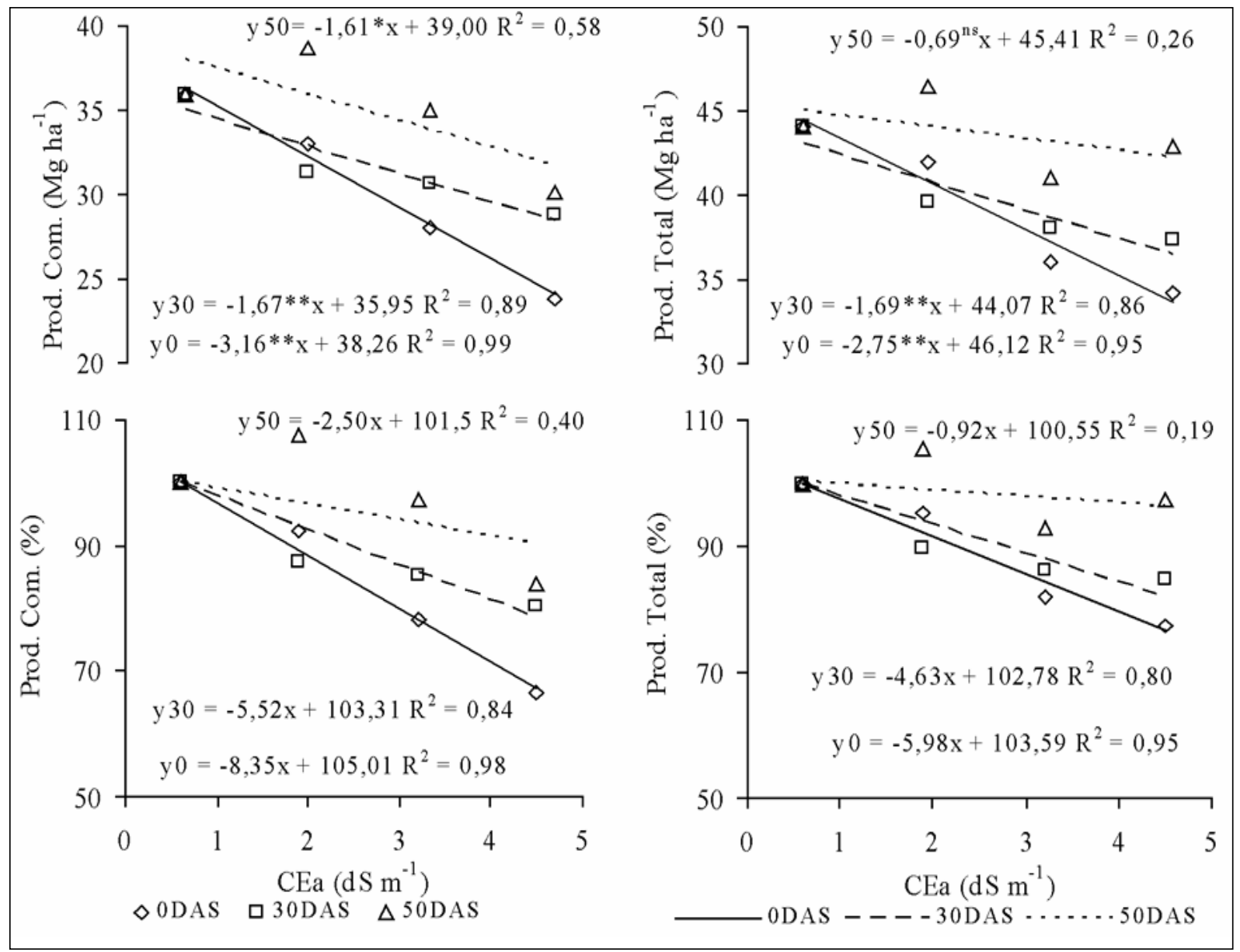

$(*)$ e $(* *)$ Parâmetros da equação significativos, respectivamente, a 0,05 e 0,01 de probabilidade e $\left({ }^{\mathrm{ns}}\right)$ não significativo a 0,05 de probabilidade, pelo teste t.

Figura 1- Produção de frutos comercial (Prod. Com.) e total (Prod. Total), em valores absolutos e relativos, do meloeiro irrigado em função de quatro níveis crescentes de salinidade da água de irrigação para 0,30 e 50 dias após semeadura (DAS), em análise conjunta dos Experimentos I e II, Mossoró, 2001 e 2002.

Tabela 4 - Valor da produção comercial (Py), custos de produção sem a água (C), custos da água por tratamento, produção comercial (Pcom) estimada e lucro da cultura do melão irrigado (Lw), em valores absolutos e relativos, em relação ao tratamento testemunha $\mathrm{T}_{1}\left(\mathrm{~S}_{1} \mathrm{~S}_{1} \mathrm{~S}_{1}\right)$. Mossoró, 2003

\begin{tabular}{|c|c|c|c|c|c|c|c|c|}
\hline \multirow[t]{2}{*}{ Tratamento } & \multirow{2}{*}{$\begin{array}{c}\text { Custo da água } \\
\mathrm{R} \$ \mathrm{ha}^{-1}\end{array}$} & \multicolumn{2}{|c|}{ Pcom estimada } & \multicolumn{2}{|c|}{ Py } & \multirow{2}{*}{$\frac{\mathrm{C}}{\mathrm{R} \$ \mathrm{ha}^{-1}}$} & \multicolumn{2}{|c|}{ Lw } \\
\hline & & $\%$ & $\mathrm{Mg} \mathrm{ha}^{-1}$ & $(\%)$ & $\mathrm{R} \$ \mathrm{Mg}^{-1}$ & & $\mathrm{R} \$ \mathrm{ha}^{-1}$ & $\%$ \\
\hline $\mathrm{T}_{1}\left(\mathrm{~S}_{1} \mathrm{~S}_{1} \mathrm{~S}_{1}\right)$ & 508,33 & 100,0 & 36,37 & 100,0 & 433,33 & 9299,44 & 5952,44 & 100,0 \\
\hline $\mathrm{T}_{5}\left(\mathrm{~S}_{1} \mathrm{~S}_{1} \mathrm{~S}_{2}\right)$ & 413,75 & 81,4 & 35,94 & 98,8 & 433,33 & 9299,44 & 5860,69 & 98,5 \\
\hline $\mathrm{T}_{6}\left(\mathrm{~S}_{1} \mathrm{~S}_{1} \mathrm{~S}_{3}\right)$ & 413,75 & 81,4 & 33,85 & 93,1 & 433,33 & 9299,44 & 4955,03 & 83,2 \\
\hline $\mathrm{T}_{7}\left(\mathrm{~S}_{1} \mathrm{~S}_{1} \mathrm{~S}_{4}\right)$ & 413,75 & 81,4 & 31,75 & 87,3 & 433,33 & 9299,44 & 4045,04 & 68,0 \\
\hline $\mathrm{T}_{8}\left(\mathrm{~S}_{1} \mathrm{~S}_{2} \mathrm{~S}_{2}\right)$ & 277,23 & 54,5 & 32,77 & 90,1 & 433,33 & 9299,44 & 4623,56 & 77,7 \\
\hline $\mathrm{T}_{9}\left(\mathrm{~S}_{1} \mathrm{~S}_{3} \mathrm{~S}_{3}\right)$ & 277,23 & 54,5 & 30,59 & 84,1 & 433,33 & 9299,44 & 3678,90 & 61,8 \\
\hline $\mathrm{T}_{10}\left(\mathrm{~S}_{1} \mathrm{~S}_{4} \mathrm{~S}_{4}\right)$ & 277,23 & 54,5 & 28,42 & 78,1 & 433,33 & 9299,44 & 2738,57 & 46,0 \\
\hline $\mathrm{T}_{2}\left(\mathrm{~S}_{2} \mathrm{~S}_{2} \mathrm{~S}_{2}\right)$ & 173,82 & 34,2 & 32,26 & 88,7 & 433,33 & 9299,44 & 4505,97 & 75,7 \\
\hline $\mathrm{T}_{3}\left(\mathrm{~S}_{3} \mathrm{~S}_{3} \mathrm{~S}_{3}\right)$ & 173,82 & 34,2 & 28,15 & 77,4 & 433,33 & 9299,44 & 2724,98 & 45,8 \\
\hline $\mathrm{T}_{4}\left(\mathrm{~S}_{4} \mathrm{~S}_{4} \mathrm{~S}_{4}\right)$ & 173,82 & 34,2 & 24,05 & 66,1 & 433,33 & 9299,44 & 948,33 & 15,9 \\
\hline
\end{tabular}




\section{APRESENTAÇÃO E AGRADECIMENTOS}

Este trabalho fez parte da Tese de Doutorado do primeiro autor, apresentada ao Curso de Doutorado em Recursos Naturais da Universidade Federal de Campina Grande (UFCG), Campina Grande, PB, Brasil.

Os autores agradecem ao Programa Avança Brasil convênio Empresa Brasileira de Pesquisa Agropecuária (EMBRAPA)/Universidade Federal Rural do Semi Árido (UFERSA) e ao Projeto Melhoria do Rendimento e da Qualidade de Frutos Produzidos no Estado do Rio Grande do Norte, convênio Financiadora de Estudos e Projetos (FINEP)/Fundação Guimarães Duque (FGD)/UFERSA, pelo financiamento do trabalho. Agradecem, também, ao Dr. Francisco Cipriano de Paula Segundo e ao Engenheiro agrônomo Aécio Fernandes Vieira, que disponibilizaram a estrutura da Fazenda Santa Júlia.

\section{REFERÊNCIAS}

ALLEN, R.G. et al. Crop evapotranspiration: guidelines for computing crop water requirements. Rome: FAO, 1998. 300p. (FAO. Irrigation and Drainage Paper, 56).

AMOR, F.M. del et al. Salinity duration and concentration affect fruit yield and quality, and growth and mineral composition of melon plants grown in perlite. HortScience, Alexandria, v.34, n.7, p.1234-1237, 1999.

BERNARDO, S. Irrigação e produtividade. I n: FARIAS, M.A. et al. (eds). Manejo de irrigação. Poços de Caldas: UFLA SBEA, 1998. Cap.1, p.117-129.

BOTÍA, P. et al. Response of eight Cucumis melo cultivars to salinity during germination and early vegetative growth. Agronomie, Paris, v.18, p.503-513, 1998.

CARMO FILHO, F. do; OLIVEIRA, O.F. Mossoró: um município do semi-árido: caracterização climática e aspecto florístico. Mossoró: ESAM, 1989. 62p. (Coleção Mossoroense, 672, série B).

CARVAJAL, M. et al. Time course of solute accumulation and water relations in muskmelon plants exposed to salt during different growth stages. Plant Science, v.138, p.103-112, 1998.

DASBERG, S.; BRESLER, E. Drip irrigation manual. Bet Dagan: IIIC, 1985. 95p.

EMBRAPA. Sistema brasileiro de classificação de solos. Centro Nacional de Pesquisas de Solos. Rio de Janeiro, 1999. 412 p.

FRANCOIS, L.E.; MAAS, E.V. Crop response and management on salt-affected soils. In: PESSARAKALI, M. (ed). Handbook of plant and crop stress. New York: Marcel Dekker, 1993. p.149-181.

FRANCO, J.A. et al. Effects of salinity on various growth stages of muskmelon cv. Revigal. Journal of Horticultural Science, v.68, n.6, p.899-904. 1993.

FRANCO, J.A. et al. Relationship between the effects of salinity on seedling leaf area and fruit yield of six muskmelon cultivars. Hortscience, Alexandria, v.32, n.4, p.642-644, 1997.

LIMA, A.A. Absorção e eficiência de utilização de nutrientes por híbridos de melão (Cucumis melo, L). 2001. 60f. Dissertação (Mestrado em Agronomia: Solos e Nutrição de Plantas) - Curso de Pós-graduação em Agronomia, Universidade Federal do Ceará.

MAAS, E.V. Crop salt tolerance. In: TANJI, K.K. (ed). Agricultural salinity assessment and management. New York: Americam Society of Civil Engineers, 1990. p.262-304.

MARTINS, L.H. Avaliação da qualidade da água nos mananciais superficiais disponíveis para irrigação na zona Oeste Potiguar. 1993. 97f. Monografia (Especialização em Irrigação e Drenagem) - Curso de Especialização em Irrigação e Drenagem, Escola Superior de Agricultura de Mossoró.

MEDEIROS, J.F. de. Qualidade da água de irrigação e evolução da salinidade nas propriedades assistidas pelo “GAT” nos Estados do RN, PB e CE. 1992.1 173f.Dissertação (Mestrado em Engenharia Agrícola: Irrigação e Drenagem) Curso de Pós-graduação em Engenharia Agrícola, Universidade Federal da Paraíba.

MENDLINGER, S.; PASTERNAK, D. Effect of time of salinization on flowering, yield and fruit quality factors in melon, Cucumis melo L. Journal of Horticultural Science, v.67, n.4, p.529-534, 1992 .

OLIVEIRA, M.; MAIA, C.E. Qualidade físico-química da água para irrigação em diferentes aqüíferos na área sedimentar do Estado do Rio Grande do Norte. Revista Brasileira de Engenharia Agrícola e Ambiental, Campina Grande, v.2, n.1, p.42-46, 1998.

PIZARRO, F.C. Riegos localizados de alta frenquencia: goteo, microaspersion, exudacion. 2.ed. Madrid: Mundi Prensa, 1990. 459p.

SUDENE. Divisão Agrológica. Mapa exploratório: reconhecimento de solos do Estado do Rio Grande do Norte. Map ed. 75 x 85cm, Escala 1:500.000, 1968. (Relatório anual).

VIVANCOS, A.D. Fertirrigacion. 2.ed. Madri: Mundi-Prensa, 1996. 233p 